\title{
Physical Needs of Long-term Cancer Patients
}

\author{
PAOLO TRALONGO ${ }^{1}$, MARIA GIOVANNA PESCARENICO ${ }^{2}$, ANTONELLA SURBONE $^{3}$, \\ SEBASTIANO BORDONARO ${ }^{1}$, MASSIMILIANO BERRETTA ${ }^{4}$ and ANNAMARIA DI MARI ${ }^{1}$ \\ ${ }^{1}$ UOC Medical Oncology, Umberto I hospital, Syracuse, Italy; \\ ${ }^{2}$ Medical Oncology, Di Vaio Hospital, Fidenza USL, Parma, Italy; \\ ${ }^{3}$ Department of Medicine, New York University Medical School, \\ Division of Medical Oncology, New York, NY, U.S.A.; \\ ${ }^{4}$ Medical Oncology Department, CRO, Aviano, Italy
}

\begin{abstract}
The enormous success in the therapeutic area of oncology has allowed achieving a number of long-term survival patients unthinkable until a few decades ago. The number of cancer survivors in the world has, in fact, almost tripled in the last decade alone. Anticancer therapies, including those of the latest generation, aimed at targeting also the chronicity of the disease, are not free from sideeffects, especially when used in the long term. This scenario should lead to development of follow-up programs with the purpose of assessing long-term effects related to cancer treatments, in addition to the early detection of any relapse or a second tumor. Oncologists who take care of cancer survivors cannot ignore these effects; it is, therefore, essential to start a program of prevention and treatment of these sequelae, to meet patients' health needs.
\end{abstract}

As a result of improved screening programs, therapeutic advances and population aging, long-term survival is increasingly common in the path of patients with a cancer diagnosis (1). While there has been an increase in the number of cancer diagnosis, also the number of cancer survivors has also increased.

In recent decades, the number of patients with a history of cancer in Italy has increased from about 2 million in 2006 to over 3 million in 2016 (2). In 2020, 4.5 million cancer patients are expected. The Italian data of cancer survivors are

This article is freely accessible online.

Correspondence to: Paolo Tralongo, UOC Medical Oncology, Umberto I Hospital, Via Testaferrata n.1, 96100, Syracuse, Italy. Tel: +393358087509, Fax: +39 0931724464, e-mail: tralongo@raosr.it

Key Words: Survivorship, cancer, treatment, toxicity, review. in accordance with the rest of the world: the number of cancer survivor in the US, for example, has increased from 3 million in 1970 to about 15 million survivors in 2012, representing $5 \%$ of the total population.

Oncologists must face a new scenario with new drugs that convert the metastatic disease to chronic and adjuvant treatments that have increased survival with, however, different and also unusual side-effects; therefore, early detection can aid avoiding the subsequent comorbidity and allowing diagnosis of early recurrence or second cancers. There are an increasing number of people of any age, health and social status that need to return to an active life, despite cancer. The institutions and clinicians need to respond to the needs of these patients who may live a long time with a disease and claim the right to recover a satisfactory quality of life (QoL). Due to the differences in defining cancer survivors in different countries (3), or identifying long-term cancer survivors, one must find a common language and plan appropriate models of care, rehabilitation and communication $(4,5)$ (Table I).

A quarter of Italian long-term cancer survivors can be considered cured (6). Yet, the improved cancer survival rates reached in recent years have medical and psychosocial implications: from late side-effects of cancer treatment that may impair the QoL to major sequaele to the risk of relapse and metachronous cancer (7). Several treatments, in fact, may be associated with various complications ranging from minor to serious and, occasionally, fatal. In addition, the increasing age of survivors is accompanied by the potential development of other diseases and multi-morbidity. Any related damaging treatment effects may, finally, increase the incidence of comorbidity, frailty and premature mortality $(8,9)$.

The national comprehensive cancer network (NCCN) guideline of 2017 defined the standards for survivorship care (Table II) that include not only the prevention and surveillance of new and recurrent cancer but also the prevention and 
Table I. Category of cancer survivors (5).

\begin{tabular}{ll}
\hline Category & Description \\
\hline Acute & Patients/survivors at first diagnosis or relapse who require acute intervention \\
Chronic & Chronic patients/survivors with cancer that slowly progressed or alternated between phases of \\
& remission and relapse, often accompanied by acceptable quality of life \\
Long-term & Patients/survivors in clinical remission for long periods of time or for their entire life who \\
& remain at risk for distant relapse or second tumors and who potentially can experience late \\
& treatment-related medical and psychosocial sequelae \\
Cured & Disease-free patients/survivors whose cancer-specific mortality and life expectancy many \\
& years after diagnosis equal that of sex- and age-matched members of the general population
\end{tabular}

Table II. Standards for survivorship care (10).

Standards for survivorship care

Care of the cancer survivor should include:

1. Prevention of new and recurrent cancers and other late effects

2. Surveillance for cancer spread, recurrence or second cancers

3. Assessment of late psychosocial and physical effects

4. Intervention for consequences of cancer and treatment (e.g., medical problems, symptoms, psychological distress, financial and social concerns)

5. Coordination of care between primary care providers and specialists to ensure that all survivors' health needs are met

6. Survivorship care planning:

$\diamond$ Develop a survivorship care plan that includes:

- Summary of treatment received

- Information regarding follow-up care and surveillance recommendations

- Information on post-treatment needs, including information regarding treatment-related effects and health risks when possible

- Delineation regarding roles of oncologists and primary care physicians, as well as timing of transfer of care if appropriate

- Health behavior recommendations

assessment of late effects and the intervention for the consequences of cancer and treatment (10). Regarding the latter, it is possible to identify those occurring during treatment or early after treatment and can last a long time, as well as those that occur long after the end of treatment and can also be long-lasting. Late side-effects can be classified into (a) sideeffects affecting specific systems (organ alterations, endocrine abnormalities, premature aging and others), (b) functional changes (incontinence, lymphedema, ostomies, osteoporosis, arthritis and others) and, finally, (c) second malignancies (11). Early recognition of late side-effects can improve patients' QoL and even survival. The "follow-up" is the "privileged phase" to recognize and treat the needs of cancer survivorships.

This review describes accurately selected late treatmentrelated medical sequelae, their pathogenesis, management, treatments and prevention.

\section{Needs}

Cardiac. Cardiac injuries can appear at the end of chemotherapy treatment and may progress or stabilize after 12 months of treatment. Literature data $(12,13)$ report improvements that could be transient or durable with a different duration for different time. It is also possible that injuries manifest at any time in patients' life (12). Patients with cardiac function reduced within 6 months of treatment should be considered at high risk of subsequent development of late cardiac failure as cardiac failure or lethal arrhythmia may occur even 20 years after treatment exposure (13).

Cardiomyopathy, pericarditis, as well as congestive heart failure, are the most typical clinical manifestations of cardiotoxicity.

Anthracyclines are one of the agents that are often responsible for cardiotoxicity. The total dose of anthracyclines is directly related to congestive cardiomyopathy. A cumulative dose of anthracyclines greater than $300 \mathrm{mg} / \mathrm{m}^{2}$ has been associated with an increase risk of heart failure (16) and shown to be present in $5 \%$ patients after 15 years from end of treatment $(14,17)$.

In the national surgical adjuvant breast and bowel project (NSABP) B-31 trial of breast cancer patients, the rates of symptomatic heart failure after 7 years were $4 \%$ in patients treated with anthracycline-based chemotherapy and trastuzumab and $1.3 \%$ in those treated with anthracyclinebased chemotherapy alone (18). 
Table III. Main ocular side-effects and correlation with medical therapy.

\begin{tabular}{ll}
\hline Dysfunction & Drug \\
\hline Blurred vision & $\begin{array}{l}\text { Cisplatin, oxaliplatin, cyclophosphamide, isophosphamide, methotrexate, paclitaxel, capecitabine, } \\
\text { imatinib, rituximab, tamoxifen, mitomycin-c, busulphan, carmustine, fludarabine, cytosine arabinoside }\end{array}$ \\
Photophobia & Fluoropyrimidine, methotrexate, fludarabine, cytosine arabinoside, pentostatin \\
Conjunctivitis & Oxaliplatin, cyclophosphamide, isophosphamide, methotrexate, 5-fluorouracil (5-FU), docetaxel \\
Dry eye & Cyclophosphamide, imatinib, nilotinib \\
Keratitis & 5-FU, capecitabine, tamoxifen, cetuximab, erlotinib, chlorambucil cytosine arabinoside \\
Lacrimal dysfunction & Oxaliplatin, cyclophosphamide, 5-FU, docetaxel, doxorubicin, cytosine arabinoside, pentostatin, panitumumab \\
Optic neuropathy & Cisplatin, carboplatin, methotrexate, paclitaxel, vincristine, tamoxifen \\
\hline
\end{tabular}

Anthracycline cardiotoxicity is higher when the treatment is associated with human epidermal growth factor receptor (16). Cardiomyocyte human epidermal growth factor receptor 2 (HER2) is uniquely susceptible to trastuzumab (17). Trastuzumab toxicity is reversible with cessation of treatment. Cyclophosphamide administered at standard or high doses in combination with mediastinal irradiation has been associated with frequent development of cardiomyopathy. Heart exposure to radiation treatment can determine valvular damage and ischemic heart disease, especially when the dose received is $>30$ Gy and when younger patients are involved (15).

Frequently, cardiac damage is clinically manifested as left ventricular dysfunction but minimally symptomatic cardiac toxicity has also been reported (16). Biologic drugs may also be responsible for cardiotoxicity. Clinically, the most frequent manifestations are represented by acute coronary syndrome, hypertension, QT prolongation, ventricular dysfunction and heart failure. Regarding the use of anti-angiogenic drugs (bevacizumab, sunitinib, sorafenib, pazopanib, vemurafenib) the damage, in terms of pathophysiology, is often caused by the alteration of the linkings of vascular endothelial growth factor (VEGF) and platelet-derived growth factor receptor (PDGFR) with their respective ligands. The inhibition of VEGF and PDGFR can lead to a reduction in myocardial capillary density and contractile dysfunction, fibrosis and heart failure. Furthermore, cardiomyocytes can promote the release of pro-apoptotic B-cell lymphoma 2 (BCL2) factor, triggering the intrinsic apoptotic pathway activation and subsequent cell death resulting in necrosis. (19) To date, in clinical practice, any myocardial damage is monitored through the assessment of left ventricular ejection fraction. Recent data indicate that troponin measurement and especially the finding of out of range values during the chemotherapy treatment could favor the identification of patients at risk for the development of ventricular dysfunction and/or additional cardiac side-effects during follow-up (20). The use of angiotensin-converting enzyme (ACE) inhibitors (such as enalapril) has been shown to have a cardioprotective effect and could, therefore, represent a strategy for prophylaxis in some categories of patients at risk (21).

Ocular. The eye is an organ with a complex neurological network with blood vessels, muscles and skin. It is a target organ for many drugs used to treat cancer: tamoxifen is responsible for retinopathy, while fluoropyrimidine is responsible of obstruction of the lacrimal gland. Probably, the ocular side-effects will increase considering the, almost ubiquitous, distribution of the molecular sites that, unfortunately, are common targets in neoplastic population (Table III). Many cancer treatments (chemotherapy, endocrine and biologic therapy) may be responsible for ocular toxicity. Eye problems are often underestimated from the epidemiological point of view, with the literature on ocular toxicity in cancer patients being poor. By contrast, the early detection of a possible ocular side-effect would allow the complete functional recovery with a significant impact on patients' QoL. The most common side-effects are photophobia, conjunctivitis, blurred vision, cataract, lacrimal dysfunction, dry eye, keratitis and neuropathy.

Pathogenetically, eye damage is sustained by different mechanisms, some not yet fully identified. During treatment with 5-fluorouracil and other fluoropyrimidines, the damage is often caused by the obstruction of the lacrimal gland; cisplatin (CDDP) results in injury to the eye through optic neuritis or retinal ischemia (23); pemetrexed is often associated with conjunctivitis through a mechanism not identified yet; tamoxifen promotes the production of retinal crystals and posterior sub-capsular opacity, mainly responsible for retinopathy. With regard to treatment with targeted therapies, there are various ocular side-effects reported with, however, not fully known yet mechanisms. In preclinical toxicity studies, a dose of $40 \mathrm{mg} / \mathrm{kg} /$ day of gefinitib determines a corneal epithelium reversible thinning in rats and dogs after an one- to six-month treatment. In dogs treated with the higher dose of gefitinib for six months, the corneal opacification became irreversible after stopping the drug. Preclinical data are consistent with the pharmacological mechanism of action of 
gefitinib because epidermal growth factor receptor (EGFR) is highly expressed in the basal epithelial cells, conjunctival and corneal epithelium. Imatinib, used for the treatment of gastrointestinal stromal tumor (GIST), can be responsible for periorbital edema, optic neuritis and macular edema (24). Pathogenetically, the damage could be determined by inhibition of c-kit expressed on dendrocytes with a consequent increase in capillary permeability and fluid formation (25).

Neurocognitive. Even if comparisons across studies are difficult, concentration difficulties, short-term memory, difficulties in problem solving, visual memory and verbal information processing are reported in long-term survivors (26). In many cases, they are durable and disabling (27).

Brain irradiation or intrathecal methotrexate or cytarabine can be responsible of neurocognitive sequaele (28) with radionecrosis, dystrophic calcification necrotizing leukoencephalopathy and cerebellar sclerosis.

The intellectual and academic achievement deficits reported in long-term survivors of childhood cancers could be due to chemotherapy or radiotherapy damage to normal white matter, intracranial endothelial cells, as well as immunological mechanisms.

The role of systemic treatment in affecting the white matter was documented in a 2012 study of 196 women with breast cancer who had been treated with adjuvant cyclophosphamide, methotrexate and fluorouracil (CMF), a regimen commonly used in those years. The patient group was compared with a control group consisting of 1,509 women. The study showed that at 21 years of median distance from treatment completion, breast cancer survivors had frequent problems concerning learning and recent memory, while their past memory had not been affected; also, in this category of patients, increased processing difficulties, problems of inhibition and motor problems were reported (29). According to the authors, the type of side-effects was similar to that recorded in the same patients at the end of treatment, being particularly relevant in patients of more advanced age.

Metabolic syndrome. The metabolic syndrome is characterized by a reduced insulin sensitivity, obesity, dyslipidemia and hypertension. The diagnosis of metabolic syndrome represents a risk factor for cardiovascular events. Endocrine treatment of different diseases can cause weight gain and reduced insulin sensitivity. Patients with prostate cancer, who have undergone chemical or surgical orchiectomy, may develop hyperglycemia and cardiovascular events (30-32), especially patients older than 65 years, as shown in a study of 41,362 older patients recruited between 1996 and 1999 and followed until 2012, where the incidence of these events was statistically higher than in the normal population (30). In the cardiovascular events, there is a discrepancy between the results of observational studies and those of clinical trials. Some recent studies do not show a risk of cardiovascular events in patients with prostate cancer treated with androgen deprivation. This is probably due to methodological bias, such as limited information on the type of tumor, duration of androgen deprivation therapy (ADT), comparison of patients with prostate cancer not treated with androgen deprivation, lack of information on the medical history (31-33). The study by O'Farrell et al., however, suggested that patients with prostate cancer, who have at least two cardiac events within the first year of starting endocrine treatment, develop a significantly higher risk of cardiac events than those who did not have cardiac disease (30). Different hormone levels may play a role in metabolic syndrome. Low levels of serum testosterone and sex hormone binding globulin have been associated with obesity, dyslipidemia and insulin resistance and other markers of vascular damage in cross-sectional studies $(32,34)$. Patients with metabolic syndrome who receive chemotherapy, when having a high body mass index (BMI) before treatment, tend to increase their BMI during follow-up and have lower levels of total testosterone and increased urinary excretion of cortisol metabolites compared with patients without metabolic syndrome. BMI and insulin are associated with metabolic syndrome, while testosterone and urinary metabolite of cortisol are associated with BMI. Testosteronerelated increased BMI plays a role in the development of metabolic syndrome. Estrogen has, in turn, a positive effect on the lipid profile. Several studies have documented that the use of tamoxifen (selective estrogen receptor modulators (SERMs)) in postmenopausal women seems to improve the lipid profile resulting in a protective effect on obesity and reduced risk of cardiovascular events (35).

It is likely that treatment with aromatase inhibitors (AI) leads to an increase in patients' cardiovascular risk by 3-4 degrees compared to those treated with tamoxifen (36). Suppressing estrogen does not probably cause a change in blood glucose or risk of diabetes but is associated with liver function abnormalities (37). In overweight and/or obese women taking tamoxifen for chemoprevention, there is an increased presence of non-alcoholic steatohepatitis. Finally, women who have undergone bilateral oophorectomy are at risk of developing metabolic syndrome, regardless of their overall health and lifestyle habits (38).

By contrast, studies show that many women being treated for breast cancer tend to prefer a high-calorie diet reducing physical activity at the same time: especially those who are overweight at diagnosis. In breast cancer patients, however, exercise can help to change the levels of insulinemia and reduce abdominal obesity; both elements observed in the metabolic syndrome.

A healthy lifestyle for these patients should include close monitoring of lipids, liver enzymes and glicemic levels to ensure early intervention. Emerging data also suggest that metformin may have beneficial effects in the prevention of 
metabolic consequences of androgen deprivation in patients not yet diabetic (39).

Thyroid. Radiation treatment to the neck is responsible for thyroid impairment (40). Iodine contrast agents may be associated with acute thyroid side-effects, including hyperthyroidism in the presence of nodules or mild Graves' disease or transient hypothyroidism in Hashimoto's thyroiditis patients. Cytotoxic agents are rarely associated with abnormalities of the thyroid gland in the absence of irradiation. However, they can sensitize the thyroid effects of concomitant radiotherapy, increasing the risk of hypothyroidism. Even immunotherapies and targeted therapies can lead to side-effects as the thyroid is a biomolecular target of various drugs. Considering the genetics of thyroid cancer, it is clear how many specific biological drugs are paradoxically responsible for thyroid toxicity. The mechanism of tyrosine kinase inhibitors (TKIs)induced hypothyroidism is unclear. Thyroid dysfunction is likely related to effects of TKIs on vascular activity. TKIs may reduce thyroid blood flow, determine transient ischemic damage and, consequently, thyrotoxicosis. When the decrease in blood flow occurs more slowly, thyroid dysfunction is more gradual and can result in hypothyroidism. Thyroid cells express VEGF mRNA and $V E G F R$, while studies on mice have shown glandular capillary regression exposure with TKIs (41). It is possible that thalidomide and lenalidomide compromise the flow of blood to the thyroid similarly to that suggested for TKIs. In a 2002 study, $20 \%$ of participants who received thalidomide for multiple myeloma has developed an increase of thyroidstimulating hormone (TSH) to $5 \mathrm{mUI} / 1$, while $7 \%$ had a $\mathrm{TSH}$ greater than $10 \mathrm{mUI} / \mathrm{l}$ (42). Hypothyroidism occurs mostly 1-6 months after initiation of treatment. In humans, two recent cases have shown a reduction in thyroid volume and vascularization with rapid recovery after discontinuation of sunitinib. (43). Hypothyroidism is the most common (9095\%) non-malignant late effect involving the thyroid gland, although hyperthyroidism and thyroid cancer have also been reported (44). Many symptoms of hypothyroidism, such as fatigue and constipation, are common in cancer patients and it may be difficult to attribute them to the tumor or antineoplastic treatments received or to drugs used to control symptoms. Unrecognized hypothyroidism or thyrotoxicosis can affect the metabolism of other drugs. Finally, although rare, thyroid dysfunction can cause complications, such as myxedema, coma and heart failure. In case of thyrotoxicosis, symptoms include palpitations, weight loss, heat intolerance, frequent bowel movements, tremors, proximal muscle weakness, tachycardia, insomnia, irritability, and fever. In the case of hypothyroidism, the symptoms include increased fatigue, weight gain, dry skin or hair, brittle nails, constipation, bradycardia and hypothermia.
Peripheral neuropathy. Several drugs can cause peripheral neuropathy (45) either as an acute side-effect, for example with Leber's hereditary optic neuropathy (LHOP), or dosedependent. This functional alteration is very important and includes dysesthesia and reduced sensitivity of the extremities leading, in some cases, to considerably reduced functional activity; this consequence affects particularly older patients who may experience loss of autonomy (46). Patients report various sensory abnormalities, including paresthesia, dysesthesia, tingling, itch, pain (constrictive, throbbing, sharp, button). Sensory deficits can lead to ataxia and gait disturbance. More rarely, patients report Lhermitte's phenomenon, which is characterized by itching, Raynaud's phenomenon and muscle aches. Myalgias have been reported in the course of treatment with gemcitabine and taxanes. Common symptoms are various types of peripheral neuropathy, twitching, disturbances of smell and taste, vestibular and sensory disturbances and Raynaud's syndrome. There are predisposing factors that must be taken into account when considering a treatment with these drugs and proposing it to cancer patients (elderly age, diabetes, alcohol abuse, prior history of neuropathies or use of other neurotoxic treatments for cancer or other diseases).

The mechanisms involved in peripheral neuropathy result from the inflammatory response with production of cytokines that can sensitize peripheral nociceptive afferents. In particular, antineoplastic agents act on ion channels (sodium, potassium, calcium) and N-methyl-D-aspartate (NMDA) receptors of ganglion roots membrane and/or spinal cord's dorsal horn neurons, altering the intracellular calcium levels and promoting pathways that trigger neuropathic pain (47) (Table IV).

Osteoporosis. Bone remodeling is a dynamic process that refers to the balance between the formation of skeletal matrix supported by osteoblasts and resorption by osteoclasts. The latter have hematopoietic origin and differentiate into multinucleated giant cells through signaling pathways that include cytokines, growth factors and hormones (48).

Osteoprotegerin (OPG) plays a key role: secreted by osteoblasts, osteoprotegerin acts as a receptor for receptor activator of nuclear factor-kappaB ligand (RANKL), inhibiting the activation of osteoclasts. Estrogens (and androgens) increase the secretion of OPG by osteoblasts, decrease RANK affinity for OPG, cause down-regulation of the expression of RANKL on osteoclasts and bone resorption. When estrogen or androgen levels decrease physiologically during menopause or for the iatrogenic effect of hormonal therapy or chemotherapy, the affinity of RANKL for OPG decreases, causing increased bone resorption. Other factors that influence the affinity of RANKL for OPG are parathyroid hormone (PTH), insulinlike growth factor 1 (IGF-1) and inflammatory cytokines.

Osteoporosis not only correlates to an endocrine abnormality but also to lifestyle, including alcohol 
Table IV. Possible mechanisms involved in the development of neuropathic pain induced by chemotherapeutic agents (48).

\begin{tabular}{lcc}
\hline S. No & Targets & Chemotherapeutic agents \\
\hline 1. & $\begin{array}{c}\text { Loss of intra-epidermal } \\
\text { nerve fibers } \\
\text { Mitochondrial changes }\end{array}$ & Vincristine, paclitaxel \\
2. & Calcium & $\begin{array}{c}\text { Pincristine, bortezomib, } \\
\text { cisplatin }\end{array}$ \\
3. & Sodium channels & Oxaliplatin, paclitaxel vincristine \\
4. & Potassium channels & Oxaliplatin
\end{tabular}
6. Transient receptor potential
vanilloid (TRPV)

7.

8.

9.

10.

N-methyl-D-aspartate
(NMDA) receptors
Neuropeptides

14.

15. Nitric oxide (NO)

Mitogen-activated protein kinase 5-hydroxytryptamine (5-HT) receptors Protein kinase C (PKC) NMDA) receptors
Cisplatin, oxaliplatin, paclitaxel

Vincristine, paclitaxel, bortezomib

Oxaliplatin, paclitaxel, vincristine, bortezomib

Paclitaxel, vincristine, oxaliplatin

Cisplatin, oxaliplatin

Cisplatin, paclitaxel and bortezomib, oxaliplatin

Paclitaxel, cispaltin

Vincristine, oxaliplatin

Vincristine

Oxaliplatin
Mechanisms

- Loss of warm and cool specific A and C fibers leading to hot and cold allodynia

- Swollen and vacuolated mitochondria in axons

- Opening of mitochondrial permeability transition pores (mPTPs) and release of intracellular

calcium, activation of caspase and apoptotic pathway

- Increased expression of subunits in dorsal horn and dorsal root ganglion (DRG)

- Increase in cytosolic calcium from extracellular (by channels) and intracellular stores from mitochondria

- Increase in $\mathrm{Na}^{+}$current in DRG predisposing to paresthesia and fasciculations

- Decreased expression of two-pore domain Weak Inward rectifying potassium channel (TWIK)-related potassium channel-1(TREK1),

TWIK-related arachidonic acid-stimulated potassium channel (TRAAK) type of inhibitory channels and increased expression of pro-excitatory channels, hyperpolarization-activated cyclic nucleotide-gated channels (HCNs)

- Up-regulation of TRPV1, transient receptor potential ankyrin 1 (TRPA1), transient receptor potential melastatin 8 (TRPM8), TRPV4 in DRG neurons leading to hyper-responsiveness of nociceptors - Increase in number of Langerhans cells (LC) on skin

- Increased release of tumor necrosis factor (TNF)-alpha and interleukin (IL)-1, IL-6 and nitric oxide (NO) from glial cells, macrophages and LC cells

- Hyper-sensitization of primary sensory afferents

- Reactive oxygen species (ROS) in DRG neurons

- Free radicals generation is secondary to increase in cytosolic calcium producing neuronal cytotoxicity

- Activation of calcium-activated proteases calpains and caspases in DRG neurons

- Initiation of apoptotic cascade leading to neuronal cytotoxicity

- Activation of p38 and extracellular signal-regulated kinase (ERK) $1 / 2$ in DRG neurons along with down-regulation of JNK/Sapk

- Dual role of ERK1/2 depending on the cellular stimulation

- NMDA receptor antagonists antagonize anticancer agents'-induced neuropathic pain - Increase in neuropeptide Y (NPY), substance P along with alteration in calcitonin gene-related peptide (CGRP) and somatostatin in DRG neurons

- Dysfunction of the spinal NO/cyclic guanosine monophosphate (cGMP) pathway

- Increase in nitric oxide synthase (NOS), particularly, neuronal NOS (nNOS) in spinal dorsal horn - Increase in 5-HT2A receptors on dorsal horn and DRG neurons - Sensitization of both peripheral nociceptive fibers and spinal dorsal horn neurons

- Increased PKC activity in supra-spinal regions

- Up-regulation of gamma/epsilon isoforms of PKC within thalamus and periaqueductal area 
consumption, smoke, corticosteroid therapy and eating habits, such as a diet low in calcium (at times consequent to treatment of gastric or other cancers). For example, the overall prevalence of osteoporosis in Korean patients suffering from gastric cancer over the age of 50 years is $39.6 \% ; 29.8 \%$ for men and $54.5 \%$ for women (49).

Skin toxicity. Skin toxicity is also a frequent side-effect. It is determined and related mainly with the activity of several drugs in bio-molecular target (anti-EGFR, anti-HER2, anti-VEGFR). The skin effects, although not a risk to life for the patients, can be huge to the point of compromising their QoL. This may lead to reducing the dosage of the drug or even discontinuing the treatment, thus putting at risk the effectiveness and outcome of cancer therapy. The pathophysiological mechanism underlying these effects on the skin is correlated with the direct action of these drugs in the skin cells and, mainly, at the level of the hair follicle where keratinocytes are present in high concentration; EGFR inhibition in proliferation of undifferentiated keratinocytes in the basal layer indeed induces growth arrest and their premature differentiation. The release of chemo-tactical factors (CXCLs, CCLS) and the recruitment of leukocytes determine apoptosis, tissue damage and vasodilation (50). It, consequently, determines folliculitis, skin rash of various degrees, photosensitivity and structural changes at the level of the hair (51).

Rash is dose-dependent and occurs in $45-100 \%$ of patients. In most cases, rash is mild (grade 1-2); in only 5$18 \%$ of treated subjects, rash reaches grade three severity. This reaction consists of pruritic erythema, folliculitis and papules/pustules, mainly confined to the seborrheic areas (face, neck, shoulders, trunk) from which pus may leak, leading to crusting. Palms of the hand and soles of the feet are always spared from this type of toxicity. Rash is an early toxic event that usually appears after no more than two or three days of drug administration with a peak after two or three weeks (52). A significant decrease in the "barrier function" of the skin that is expressed also with the phenomenon of dryness (xerosis) until the skin fissures has also been reported. Finally, even nail growth can undergo changes that occur with phenomena of onychocryptosis, as a result of the reactive pyogenic granuloma.

Other common needs. Pulmonary fibrosis is a complication correlated to radiation treatments but also to chemotherapeutic agents, such as anthracycline and bleomycin (53). The clinical presentation may vary in degrees from mild to severe and symptoms may occur only during physical activity. Genitourinary alterations can occur after the use of chemotherapeutic agents causing glomerular and tubular damage, such as cisplatin, methotrexate and nitrosoureas (54). Genitourinary abnormalities in patients with prostate cancer may be clinically characterized by urinary obstruction (more frequent on the right) (55). Frequently, with the use of fluoropyrimidines, including oral ones, or radiation therapy to the abdomen and pelvis diarrhea may occur, as well as alterations in liver function indexes, which can lead to forms of toxic hepatitis and liver failure (56) (Table V).

\section{How to Identify and Treat Needs}

The main goal of cancer specialists is to optimize the quality of life of their patients by monitoring and controlling potential treatment side-effects and comorbidity. Preventing interventions must be promoted by removing, whenever possible, the causes by identifying the specific individual risk(s) of each patient with reference to each proper therapy for the type of cancer concerned. Though it is not always possible to modify a regimen according to individual patients' characteristics, it is always helpful to know these characteristics before initiating any cancer therapy. In the following section, simple measures to implement an effective management of side-effects will be analyzed (Table VI).

- Cardiac toxicity in clinical practice should be monitored through cardiologic examination, including echocardiography, which can provide descriptive data on the heart's contractile function and left ventricular ejection fraction, and electrocardiographic examination to highlight alterations of electrical conduction (hypertension, congestive heart failure, QT prolongation). The management of ventricular dysfunction and heart failure in patients treated with antiangiogenic drugs includes echocardiographic monitoring, evaluation of New York heart association (NYHA) class and hypertension. In some patients, for example those treated with trastuzumab, it is possible to resume treatment if there is a sufficient functional recovery of the contractile activity (Figure 1) (57). In patients at risk for advanced age, heart disease, kidney disease, diabetes or obesity, certain studies have shown that elevated values of serum troponin during chemotherapy may identify myocardial damage in a subclinical stage and cardio-protective drug ACE inhibitors (such as enalapril) may represent a prophylactic strategy (58). Some relatively simple interventions are crucial to prevent cardiac damage from modifying patients' lifestyle through a healthy diet and elimination of alcohol and smoking, thus encouraging moderate physical activity and periodic clinical evaluation, including blood tests with coagulation and lipid profile, as part of regular follow-up. Aerobic exercise is an effective non-pharmacological therapy in attenuating doxorubicin (DOX)-induced cardiotoxicity. Aerobic exercise is well-documented to improve systolic and diastolic function and attenuate pathological cardiac remodeling in patients with heart failure. Preclinical data suggest several pathways to mediate the protective properties of the exercise: enhancing endogenous antioxidant protective 
Table V. Common side-effects and etiopathogenesis.

\begin{tabular}{|c|c|}
\hline Type of side-effects & Drugs $\Rightarrow$ etiopathogenesis \\
\hline $\begin{array}{l}\text { Ventricular dysfunction } \\
\text { Heart failure } \\
\text { Pericarditis } \\
\text { Arrhythmias }\end{array}$ & $\begin{array}{l}\text { Anthracyclines, trastuzumab, vinorelbine, fluoropyrimidine } \Rightarrow \\
\text { myocardial damage sometimes dose-dependent and reversible }\end{array}$ \\
\hline $\begin{array}{l}\text { Arrhythmias } \\
\text { Photophobia } \\
\text { Dry eye } \\
\text { Keratitis } \\
\text { Cataract } \\
\text { Lacrimal disorders }\end{array}$ & $\begin{array}{l}\text { Cisplatin, pemetrexed, gemcitabin, tamoxifen, TKI } \Rightarrow \\
\text { etiopathogenetic mechanism(s) not always known }\end{array}$ \\
\hline $\begin{array}{l}\text { Disorders of memory and learning } \\
\text { Processing difficulties } \\
\text { Motor disorders } \\
\text { Language disorders }\end{array}$ & $\begin{array}{l}\text { Radiotherapy, methotrexate, citarabine, fluorouracil, cyclophosphamide } \Rightarrow \\
\text { cerebellar sclerosis, dystrophic calcification, necrosis }\end{array}$ \\
\hline Metabolic syndrome & $\begin{array}{l}\text { Endocrine and chemotherapy treatments } \Rightarrow \text { increase in body mass index (BMI), } \\
\text { hyperinsulinemia, abnormal estrogen and testosterone levels }\end{array}$ \\
\hline $\begin{array}{l}\text { Hyper/Hypothyroidism } \\
\text { Thyrotoxicosis }\end{array}$ & $\begin{array}{l}\text { Use of iodinated contrast agents, radiation therapy, chemotherapy, targeted therapy } \Rightarrow \\
\text { etiopathogenetic mechanisms not fully known, probable alteration of thyroid vascularity }\end{array}$ \\
\hline Dysesthesia, paresthesia, pain, myalgia, ataxia & Taxol, vinorelbin, cisplatin \\
\hline Osteoporosis & $\begin{array}{l}\text { Endocrine treatments and chemotherapy, steroid therapy, alcohol, smoking and } \\
\text { diet low in calcium } \Rightarrow \text { Osteoprotegerin }(\mathrm{OPG}) \text {, receptor activator of nuclear } \\
\text { factor-kappaB ligand (RANKL), parathyroid hormone (PTH), insulin-like growth } \\
\text { factor } 1 \text { (IGF-1) interaction(s) }\end{array}$ \\
\hline $\begin{array}{l}\text { Skin reaction, photosensitivity, xerosis, } \\
\text { change in the nail }\end{array}$ & $\begin{array}{l}\text { Anti-epidermal growth factor receptor (EGFR) } \Rightarrow \text { growth arrest and premature } \\
\text { differentiation of basal keratinocytes, with release of chemo-tactical factors (CXCLs, CCLS) } \\
\text { and recruitment of leukocytes that determine apoptosis, tissue damage and vasodilation }\end{array}$ \\
\hline Pulmonary fibrosis & $\begin{array}{l}\text { Radiation therapy and chemotherapy (bleomycin, anthracyclines) sometimes } \\
\text { dose-dependent and reversible }\end{array}$ \\
\hline Genitourinary disorders & $\begin{array}{l}\text { Chemotherapy (platinum and derivatives, methotrexate, nitrosoureas) } \Rightarrow \\
\text { glomerular and tubular insult, urinary obstruction in pieces with prostate carcinoma }\end{array}$ \\
\hline Gastrointestinal disorders (diarrhea) & $\begin{array}{l}\text { Radiation therapy, chemotherapy, targeted therapy, liver toxicity } \Rightarrow \\
\text { mucosa insult sometimes dose-dependent and reversible }\end{array}$ \\
\hline
\end{tabular}

machinery, regulating pro-apoptotic signaling, limiting myocyte turn-over following DOX, preventing calcium overload in the myocyte (59). Furthermore, emerging evidence indicates that aerobic exercise is a non-toxic, pleiotropic therapy that affects diverse cardiac signaling pathways implicated in the cardiotoxicity induced by antiHER2 and anti-angiogenic therapy (60).

- Ocular toxicity should be monitored clinically. A preliminary evaluation (before any cancer treatment) helps identify categories at risk with subclinical alterations requiring close monitoring. An initial screening program, therefore, is essential for a better assessment of the effects of treatments. To date, there are only few studies available aiming at orientation of prophylactic interventions. Several studies have evaluated different approaches for the prevention of conjunctivitis induced by anti-metabolites. A randomized study by Matteucci et al. evaluated the effectiveness of steroids in combination with topical anti-inflammatory drugs (dexamethasone + diclofenac) for prophylaxis of conjunctivitis induced by high-dose cytosine arabinoside. The results show that the combination of diclofenac and dexamethasone compared to dexamethasone alone reduces significantly conjunctivitis induced by these drugs (13\% vs. $45 \%$ incidence, respectively; $p<0.09$ ) (61). It is, therefore, mandatory to refer patients to an eye specialist as soon as symptoms of conjunctivitis related to treatment appear.

- The management of cognitive impairment involves cognitive rehabilitation, adequate sleep hygiene, physical and mental activity, drug therapy (erythropoietin (EPO), antioxidants, methylphenidate, donepezil).

- Metabolic syndrome should be monitored through regular follow-up, including clinical examination and blood tests. Clinical examination should include evaluation of BMI, waist circumference measurement, detection of pressure parameters, glucose dosage, insulin and cholesterol. Patients should be encouraged to perform physical activity, even moderate, and follow a healthy diet. Finally, groups at risk for the use of therapies that interfere with insulin and BMI 
Table VI. Specific measures to evaluate late effects and ancillary interventions.

\begin{tabular}{|c|c|}
\hline Content of clinic visit & Suggested evaluative procedures and ancillary actions \\
\hline $\begin{array}{l}\text { Growth: includes issues, such as } \\
\text { short stature, scoliosis, hypoplasia } \\
\text { Cardiac }\end{array}$ & $\begin{array}{l}\text { Monitor growth (growth curve); sitting height; parental heights, nutritional status/diet; evaluate scoliosis, } \\
\text { bone age, growth hormone assays, thyroid function; endocrinologist's consultation; orthopedic consultation } \\
\text { Electrocardiogram, echo, afterload reduction, cardiologist's consultation. } \\
\text { Counseling against isometric exercises if high risk, advise ob/gyn risk of cardiac failure in pregnancy }\end{array}$ \\
\hline Neurocognitive & $\begin{array}{l}\text { History and exam. } \\
\text { Communicate: school, family, special education. } \\
\text { Compensatory remediation techniques. Neuropsychology consultation; computed tomography or } \\
\text { magnetic resonance imaging; cerebrospinal fluid; basic myelin protein. } \\
\text { Written instructions, appointment cards }\end{array}$ \\
\hline Neuropathy & $\begin{array}{l}\text { History/exam: neurologic exam, sensory changes hands/feet, paresthesias, bladder, gait, vision, muscle strength } \\
\text { Neurologic consultation }\end{array}$ \\
\hline Gonadal toxicity & $\begin{array}{l}\text { History for primary } v s \text {. secondary dysfunction, gonadal function (menstrual cycle, pubertal } \\
\text { development/delay, libido); hormone therapy; interventions (bromocriptine) } \\
\text { Premature menopause: hormone replacement unless contraindicated, Dual-energy X-ray absorptiometry } \\
\text { scans for osteoporosis; calcium. Endocrinological consultation. Reproductive technologies }\end{array}$ \\
\hline
\end{tabular}

(AIs, androgen deprivation) should be identified. A wealth of evidence has demonstrated that regular physical activity is associated with lower risk of death, all-cause mortality, cancer recurrence and several chronic diseases, including type 2 diabetes and cardiovascular disease, common comorbid conditions in people who have survived cancer. In addition, several reviews and meta-analyses have demonstrated that physical activity is associated with a lower risk of death, allcause mortality and cancer recurrence, particularly among breast and colorectal cancer survivors (62). Although cancer survivors may have high levels of interest in lifestyle interventions, they may have special needs (e.g., fatigue, incontinence, lymphedema, food intolerances or digestive disorders, long-term addiction to tobacco products) that must be taken into account if attempts to promote exercise, healthful dietary change and smoking cessation are to be successful (63). Moreover, there is evidence that cancer survivors tend to reduce physical activity (64): sedentary behavior is independently associated with chronic diseaserelated risk factors, such as central adiposity, elevated blood glucose and insulin, and other markers of cardiovascular disease risk in healthy adults (65) (Table VII).

- Thyroid toxicity can manifest through a variety of symptoms that may be initially subtle. At the first signs of thyroid dysfunction, or when expected as potential consequence of cancer treatment, blood tests should be obtained and the patient referred to a specialist who will assess the function and structure of the gland through further laboratory tests and instrumental measurements (ultrasound, scintigraphy). Identifying patients for potential thyroid toxicity with these tests is of fundamental importance.

- Neurotoxicity can present with different clinical features, including reversible encephalopathy, aphasia, confusion, blindness, coma, parkinsonism and peripheral neurotoxicity. Basic neurological examination of every patient is needed and numerous tests are available to evaluate and quantify the damage responsible for each clinical manifestation. Symptoms often result in functional impairment. As not to impact on the QoL and also compromise the simplest daily activities, it is, therefore, necessary to identify patients with higher risk of neurotoxicity, such as those of more advanced age or suffering from diabetes or pre-existing neuropathies or previously treated with neurotoxic drugs or those with history of alcohol or substance abuse. Regarding the management of chemotherapy-induced peripheral neuropathy, it is possible to use duloxetine, although with moderate strength of recommendation and intermediate level of evidence (66).

- Osteoporosis is the most common pathology of the skeletal system characterized by progressive decrease in bone mass and deterioration of bone structure. As well as for hypertension and hypercholesterolemia, osteoporosis is a silent disease that may progress for several years before being diagnosed when a fracture occurs. Therefore, in the evaluation of patients it is essential to identify different risk groups: elderly patients who physiologically undergo a progressive decrease in bone mineralization, women undergoing endocrine treatment for breast cancer, men receiving hormonal therapy for prostate cancer or patients on chronic treatment with glucocorticoids. The most sensitive test to quantify bone density is bone densitometry. Among other tests measuring accurately bone density there are ultrasonography, computed tomography (CT), single or dual photon absorptiometry (DPA) and dual-energy X-ray absorptiometry (DXA) using an X-ray source instead of a radioactive isotope. Blood tests allow a differential diagnosis between primary and secondary forms. Interventions, in case 


\section{Management of left ventricular dysfunction and heart failure induced by anti-angiogenetic therapies}

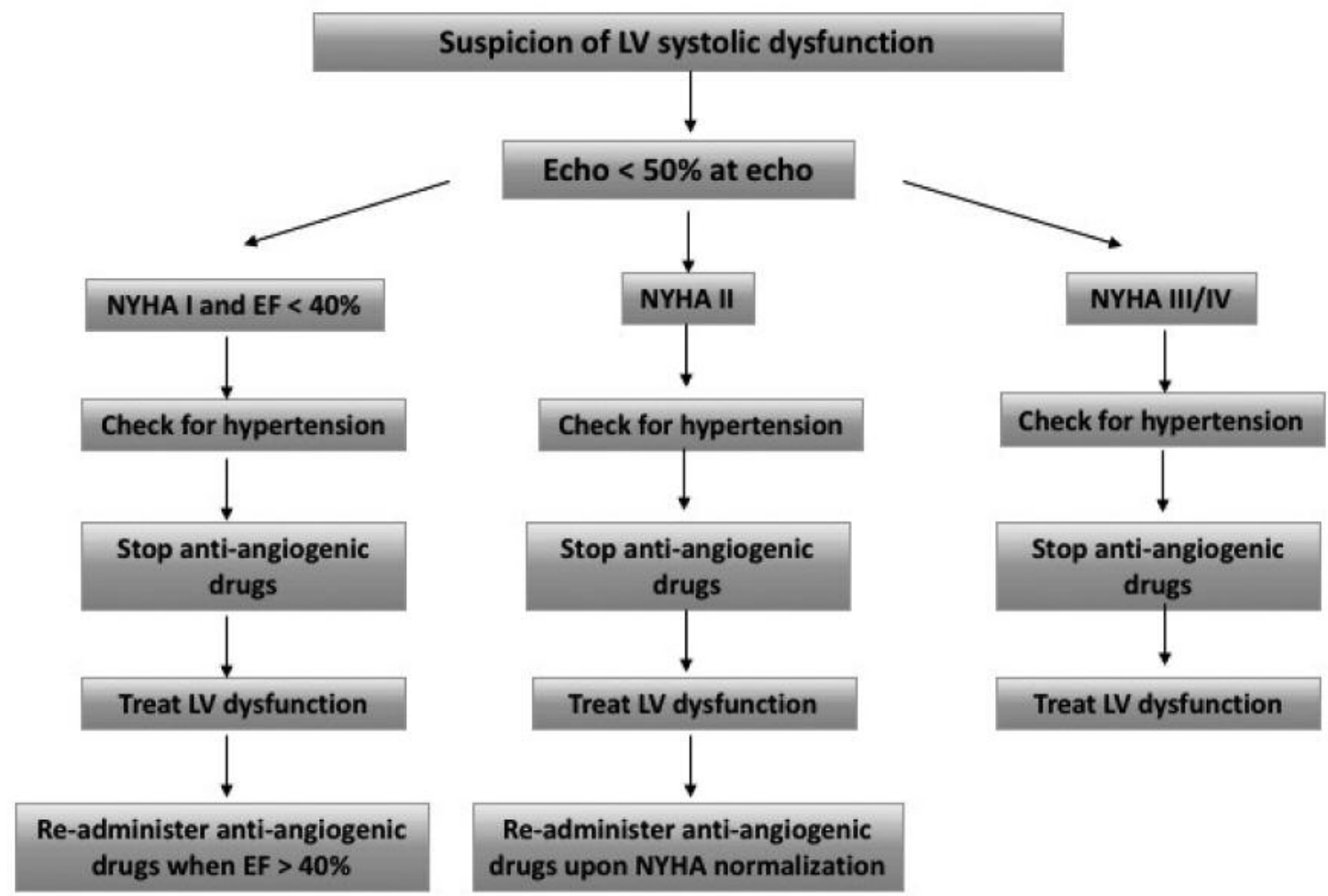

Figure 1. Management of cardiac dysfunction induced by anti-angiogenic therapies (22). NYHA, New York heart association; LV, left ventricular; $E F$, ejection fraction.

of osteopenia or frank osteoporosis, should include adequate intake of calcium and vitamin $\mathrm{D}$, implementation of physical activity, limiting alcohol consumption, healthy diet, limited use of tobacco and the use of bisphosphonates, either intravenously or in oral form. A recent study showed that treatment with oral clodronate, as a preventive measure for three years, significantly reduced the incidence of osteoporosis in patients who received chemotherapy in the adjuvant setting or anti-estrogens for breast cancer. The reduction of osteoporosis was still evident seven years after cessation of treatment with clodronate. Finally, it is important to evaluate additional risk factors, such as chronic intake of corticosteroids, AIs, antidepressants, selective serotonin reuptake inhibitors (SSRIs), certain anticonvulsants, antacids, proton pump inhibitors and aluminum antacids.

- The management of cutaneous side-effects induced by EGFR inhibitors has already been investigated in several clinical studies $(67,68)$. All authors agreed on the role of prevention and treatment of rash without necessarily resorting to dose reduction that could compromise the antitumor effect. On the basis of the published literature on the subject and on the experience of clinical oncologists and dermatologists, this study suggests the following guidelines for rash management:

1. Mild skin lesions (G1). Maintaining therapy with EGFR inhibitors to standard dosing and treating injuries using topical medications. Since this type of dermatitis is of inflammatory origin, anti-inflammatory drugs are to be preferred.

Topical corticosteroids: They act in the short-term (7-10 days). It is recommended not to prepare with fatty vehicles to avoid occlusion.

Topical erythromycin and clindamycin: Antibiotics having high anti-inflammatory activity in acne. The pharmacological effect is observed, in general, in 4-6 weeks.

Azelaic acid: The pharmacological effect is observed, in general, in 4-6 weeks. 
Table VII. Interventions to promote health and lifestyles.

Interventions

Avoid sedentary lifestyle and resume physical activity as soon as possible

For patients aged 18 to 65 : practice at least 150 minutes of moderate activity and 75 minutes of vigorous activity per week

Dedicate at least two days per week to work-out

Healthy eating

Avoid smoking and alcohol consumption

Metronidazole: The pharmacological effect is observed, in general, in 4-6 weeks.

2. Moderate lesions (G2). It is appropriate to discontinue therapy for a week and then resume a full dose. If the injury improves or reduces in degree spontaneously or with treatment, it is useful to continue treatment without dose reduction; if the injury does not improve despite topical and/or systemic therapy, reduce EGFR inhibitor dose by $30 \%$; if the injury worsens, consider discontinuation of treatment and adopt grade 3 and 4 lesion regimen.

3. Severe lesions (G3-4). Depending on the severity of the skin rash, it is appropriate to consider the possibility of reducing the dose or, in severe cases, to suspend treatment. For the treatment of skin rash we used in clinical practice the following medications:

Systemic corticosteroids: Rapid anti-inflammatory effect. In case of long-term therapy (over 3-4 weeks), the use of corticosteroids may cause rosacea-forming eruptions with characteristics similar to skin rash. According to experts, should be taken caution in using these drugs, especially for the rebound effects that might occur after their suspension.

Tetracyclines (minocycline, doxycycline, tetracycline base): With an amply demonstrated anti-inflammatory effect (69). They have been long and successfully used in the treatment of acne, although they have a slower action. As for their antibiotic activity, it should be noted that due to the extensive use of these drugs, both humans and animals have developed a widespread resistance to $S$. aureus and another pathogenic Gram-positive strain (Streptococcus).

Targeted antibiotics: A systemic antibiotic therapy can be administered on material taken from pustules on infected secondary injuries.

In clinical practice, other drugs are sometimes used for which there is not, to date, definitive evidence. These drugs include sulfur salicylic creams, gentamicin with or without cortisone, topical in particularly serious cases, oily emulsions, neutral soaps. In case of paronychia, also
Table VIII. Follow-up goals.

Guidelines

Identifying recurrent disease

Identifying acute and late iatrogenic side-effects

Identifying psychosocial distress

Adherence to follow-up

Screening programs (diagnosis of second cancers)

aluminum salts or chlorhexidine are used. Topical retinoids should be avoided since they can be locally irritating and do not show any advantage over the other aforementioned products.

\section{Follow-up and Prevention Intervention}

Due to the complexity of different clinical cases, we need to monitor patients through specific surveillance programs for timely monitoring potential late side-effects of cancer treatments and early detection of any disease recurrence or synchronous or metachronous cancer. In addition, surveillance programs should include counseling and encouraging patients in adopting changes in their lifestyle. The choice and implementation of such measures may vary according to the characteristics of each patient, still taking into account already available guidelines for the follow-up of patients with different types and stages of cancer (Table VIII).

The aims of the follow-up are not only those to intercept a relapse but also to identify, monitor and treat any complications of previous treatments and long-term effects of care, thus promoting physical, psychological and social rehabilitation.

In addition, follow-up should also include planning of interventions for health promotion and changing incorrect lifestyles, suggesting the adoption of a healthy diet, adequate physical activity and the removal of risk factors, such as smoking or alcohol (Table IX). 
Table IX. Treatment and prophylaxis.

\begin{tabular}{ll}
\hline Side-effect & Prophylaxis \\
\hline Cardiac toxicity & Use of angiotensin-converting enzyme (ACE) inhibitors as a preventive measure \\
Ocular toxicity & Use of topical steroids and anti-inflammatory drugs, also prophylactic \\
Skin toxicity & Use of antibiotics, steroids and anti-inflammatory drugs, also prophylactic \\
Cognitive disorders & Rehabilitation, sleep hygiene, drugs \\
Metabolic syndrome & Exercise and nourishing diet to reduce insulin levels \\
Thyroid toxicity & Thyroid function indicators $\Rightarrow$ replacement therapy \\
Neurotoxicity & Identifying patients at risk $\Rightarrow$ Neuroprotective \\
Osteoporosis & Bone mineral density; avoid smoking and alcohol; prefer a diet rich in calcium, any replacement treatment \\
Pulmonary fibrosis & Pulmonary rehabilitation in case of reversibility \\
Genitourinary abnormalities & Basal and following renal function evaluation \\
& Gonadal preservation, rehabilitation \\
Gastrointestinal disorders & Prefer other drugs (carboplatin) \\
\hline
\end{tabular}

\section{Conclusion}

In the past, diagnosis of cancer was almost always associated with poor life expectancy. Due to the lack of adequate screening programs for monitoring late side-effects and promote early diagnosis of relapses or new cancers, as well as to limited therapeutic approaches, both survival and QoL of patients were poor. Today, cancer is curable and many patients may expect to become long-term or chronic cancer patients. This phenomenon is characterized by a complex spectrum of medical and psychosocial problems that need to be monitored, detected and often treated.

As a result, every cancer patient, irrespective of stage, should be tracked and monitored over time. Similarly to what happens in other chronic diseases, such as diabetes, hypertensive heart disease or chronic renal failure, each patient should be monitored for potential side-effects and late sequaele of the treatment received. All side-effects should be carefully evaluated, even those apparently not relevant; some late side-effects, in fact, may not be considered particularly serious form a medical viewpoint, yet they could result to be quite debilitating and negatively impact on patients QoL, especially when the effects are persisting for long periods. For example, a modest diarrhea (3-4 discharges per day) does not require hospitalization or specific medical treatment except symptomatic treatment; it may, however, consistently affect patients' life in their relations and daily routine.

Tools for patients' categorization and classification of late side-effects are available. It is, thus, crucial for oncologists and general practitioners to acquire specific knowledge of these instruments, to ensure the best QoL for their patients.

\section{References}

1 Grant M, Economou D, Ferrell B and Uman G: From cancer patient to cancer survivor: Lost in transition. Washington, DC, USA.The National Academies Press, 2006.

2 AIRTUM 2015. I numeri del cancro in Italia: http:// www.registritumori.it/PDF/AIOM2015/I_numeri_del_cancro_2015.pdf.

3 Surbone A, Annunziata MA, Santoro A, Tirelli U and Tralongo P: Cancer patients and survivors: Changing words or changing culture? Ann Oncol 24(10): 2468-2471, 2013.

4 Surveillance, Epidemiology, and End Results (SEER) Program. SEERStat Database: Incidence-SEER-9 regulations. Bethesda, MD, National Cancer Institute, Division of Cancer Control and Population Sciences, 2003.

5 Surbone A and Tralongo P: Categorization of cancer survivors: Why we need it. J Clin Oncol 34(28): 3372-3374, 2016.

6 Hewitt M, Rowland JH and Yancik R: Cancer survivors in the United States: Age, health, and disability. J Gerontol A Biol Sci Med Sci 58: 82-91, 2003.

7 Dal Maso L, Guzzinati S, Buzzoni C, Capocaccia R, Serraino D, Caldarella A, Dei Tos AP, Falcini F, Autelitano M, Masanotti G, Ferretti S, Tisano F, Tirelli U, Crocetti E, De Angelis R and AIRTUM Working group: Long-term survival, prevalence, and cure of cancer: A population-based estimation for 818902 Italian patients and 26 cancer types. Ann Oncol 25: 2251-2260, 2014.

8 McCabe MS, Bhatia S, Oeffinger KC, Reaman GH, Tyne C, Wollins DSn and Hudson MM: American Society of Clinical Oncology Statement: Achieving high-quality cancer survivorship care. J Clin Oncol 31(5): 631-640, 2013.

9 Valdivieso M1, Kujawa AM, Jones T and Baker LH: Cancer survivors in the United States: A review of the literature and a call action. Int J Med Sci 9(2): 163-173, 2012.

10 NCCN Guideline Version 12017 . Survivorship.

11 Aziz NM: Late effects of cancer treatments. Cancer survivorship today and tomorrow, P.A Ganz Editor, pp. 54-76, 2007, Springer. 
12 Cardinale D, Colombo A, Bacchiani G, Tedeschi I, Meroni CA, Veglia F, Civelli M, Lamantia G, Colombo N, Curigliano G, Fiorentini $\mathrm{C}$ and Cipolla CM: Early detection of anthracycline cardiotoxicity and improvement with heart failure therapy. Circulation 131(22): 1981-1988, 2015.

13 Steinherz LJ, Steinherz PG, Tan CT, Heller G and Murphy ML: Cardiac toxicity 4 to 20 years after completing anthracyline therapy. JAMA 266: 1672-1677, 1991.

14 Curigliano G, Cardinale D, Suter T, Plataniotis G, de Azambuja E, Sandri MT, Criscitiello C, Goldhirsch A, Cipolla C, Roila F and ESMO Guidelines Working Group: Cardiovascular toxicity induced by chemotherapy, targeted agents and radiotherapy: ESMO Clinical Practice Guidelines. Ann Oncol 23(Suppl 7): vii155-166, 2012.

15 Cardinale D, Colombo A, Lamantia G, Colombo N, Civelli M, De Giacomi G, Rubino M, Veglia F, Fiorentini C and Cipolla $\mathrm{CM}$ : Anthracycline-induced cardiomyopathy. Clinical relevance and response to pharmacologic therapy. J Am Coll Cardiol 55: 213-220, 2010.

16 Cardinale D, Colombo A, Torrisi R, Sandri MT, Civelli M, Salvatici M, Lamantia G, Colombo N, Cortinovis S, Dessanai MA, Nolè F, Veglia F and Cipolla CM: Trastuzumab-induced cardiotoxicity: Clinical and prognostic implications of troponin I evaluation. J Clin Oncol 28: 3910-3916, 2010.

17 Suter TM and Ewer MS: Cancer drugs and the heart: Importance and management. Eur Heart J 34: 1102-1111, 2013.

18 Romond EH, Jeong JH, Rastogi P, Swain SM, Geyer CE Jr., Ewer MS, Rathi V, Fehrenbacher L, Brufsky A, Azar CA, Flynn PJ, Zapas JL, Polikoff J, Gross HM, Biggs DD, Atkins JN, TanChiu E, Zheng P, Yothers G, Mamounas EP and Wolmark N: Seven-year follow-up assessment of cardiac function in NSABP $\mathrm{B}-31$, a randomized trial comparing doxorubicin and cyclophosphamide followed by paclitaxel (ACP) with ACP plus trastuzumab as adjuvant therapy for patients with node-positive human epidermal growth factor receptor 2-positive breast cancer. J Clin Oncol 30(31): 3792-3799, 2012.

19 Kido M, Du L, Sullivan CC, Li X, Deutsch R, Jamieson SW and Thistlethwaite PA: Hypoxia-inducible factor 1-alpha reduces infarction and attenuates progression of cardiac dysfunction after myocardial infarction in the mouse. J Am Coll Cardiol 46(11): 2116-2124, 2005.

20 Cardinale D, Sandri MT, Colombo A, Colombo N, Boeri M, Lamantia G, Civelli M, Peccatori F, Martinelli G, Fiorentini C and Cipolla CM: Prognostic value of troponin I in cardiac risk stratification of cancer patients undergoing high-dose chemotherapy. Circulation 109: 2749-2754, 2004.

21 Colombo A, Sandri MT, Salvatici M, Cipolla CM and Cardinale D: Cardiac complications of chemotherapy: Role of biomarkers Curr Treat Options Cardiovasc Med 16(6): 313, 2014.

22 Tocchetti CG, Gallucci G, Coppola C, Piscopo G, Cipresso C, Maurea C, Giudice A, Iaffaioli RV, Arra C and Maurea N: The emerging issue of cardiac dysfunction induced by antineoplastic angiogenesis inhibitors. Eur J Heart Fail 15(5): 482-489, 2013.

23 Kwan AS, Sahu A and Palexes G: Retinal ischemia with neovascularization in cisplatin related retinal toxicity. Am J Ophthalmol 141(1): 196-197, 2006.

24 Agustoni F, Platania M, Vitali M, Zilembo N, Haspinger E, Sinno V, Gallucci R, de Braud F and Garassino MC: Emerging toxicities in the treatment of non-small cell lung cancer: ocular disorders. Cancer Treat Rev 40(1): 197-203, 2014.
25 Renouf DJ, Velazquez-Martin JP, Simpson R, Siu LL and Bedard PL: Ocular toxicity of targeted therapies. J Clin Oncol 30: 32773286, 2012.

26 Foster C, Wright D, Hill H, Hopkinson J and Roffe L: Psychosocial implications of living 5 years or more following a cancer diagnosis: A systematic review of the research evidence. Eur J Cancer Care (Engl) 18: 223-247, 2009.

27 Hutchinson AD, Hosking JR, Kichenadasse G, Mattiske JK and Wilson C: Objective and subjective cognitive impairment following chemotherapy for cancer: A systematic review. Cancer Treat Rev 38(7): 926-934, 2012.

28 Tallet AV, Azria D, Barlesi F, Spano JP, Carpentier AF, Gonçalves A and Metellus P: Neurocognitive function impairment after whole brain radiotherapy for brain metastases: Actual assessment. Radiat Oncolol 7: 77, 2012.

29 Koppelmans V, Breteler MM, Boogerd W, Seynaeve C, Gundy $\mathrm{C}$ and Schagen SB: Neuropsychological performance in survivors of breast cancer more than 20 years after adjuvant chemotherapy. J Clin Oncol 30: 1080-1086, 2012.

30 O'Farrell S, Garmo H, Holmberg L, Adolfsson J, Stattin P and Van Hemelrijck M: Risk and timing of cardiovascular disease after androgen-deprivation therapy in men with prostate cancer. J Clin Oncol 33(11): 1243-1251, 2015.

31 Morote J, Orsola A, Planas J, Trilla E, Raventós CX, Cecchini L and Catalán R: Redefining clinically significant castration levels in patients with prostate cancer receiving continuous androgen deprivation therapy. J Urol 178: 1290-1295, 2007.

32 Braga-Basaria M, Dobs AS, Muller DC, Carducci MA, John M, Egan $\mathrm{J}$ and Basaria S: Metabolic syndrome in men with prostate cancer undergoing long-term androgen-deprivation therapy. J Clin Oncol 24: 3979-3983, 2006

33 Tsai HK, D'Amico AV, Sadetsky N, Chen MH and Carroll PR: Androgen deprivation therapy for localized prostate cancer and the risk of cardiovascular mortality. J Natl Cancer Inst 99: 1516$1524,2007$.

34 Smith MR, Goode M, Zietman AL, McGovern FJ, Lee H and Finkelstein JS: Bicalutamide monotherapy versus leuprolide monotherapy for prostate cancer: Effects on bone mineral density and body composition. J Clin Oncol 22: 2546-2553, 2004.

35 Chan DS, Vieira AR, Aune D, Bandera EV, Greenwood DC, McTiernan A, Navarro Rosenblatt D, Thune I, Vieira R and Norat T: Body mass index and survival in women with breast cancer-systematic literature review and meta-analysis of 82 follow-up studies. Ann Oncol 25: 1901-1914, 2014.

36 Sestak I, Distler W, Forbes JF, Dowsett M, Howell A and Cuzick $\mathrm{J}$ : Effect of body mass index on recurrences in tamoxifen and anastrozole treated women: An exploratory analysis from the ATAC trial. J Clin Oncol 28: 3411-3415, 2010.

37 Reding KW, Daling JR, Doody DR, O'Brien CA, Porter PL and Malone KE: Effect of prediagnostic alcohol consumption on survival after breast cancer in young women. Cancer Epidemiol Biomarkers Prev 17: 1988-1996, 2008.

38 Chen X, Lu W, Zheng W, Gu K, Chen Z, Zheng Y and Shu XO: Obesity and weight change in relation to breast cancer survival. Breast Cancer Res Treat 122: 823-833, 2010.

39 Bayraktar S, Hernadez-Aya LF, Lei X, Meric-Bernstam F, Litton JK, Hsu L, Hortobagyi GN and Gonzalez-Angulo AM: Effect of metformin on survival outcomes in diabetic patients with triple receptor-negative breast cancer. Cancer 118: 1202-1211, 2012. 
40 Hancock SL, McDougall IR and Constine LS: Thyroid abnormalities after therapeutic external radiation. Int J Radiat Oncol Biol Physics 31(5): 1165-1170, 1995.

41 Jazvić M, Prpić M, Jukić T, Murgić J, Jakšić B, Kust D, Prgomet A, Bolanča A and Kusić Z: Sunitinib-induced thyrotoxicosis - a not so rare entity. Anticancer Res 35(1): 481-485, 2015.

42 Rupa-Matysek J, Gil L and Komarnicki M: Thrombotic complications following the treatment of multiple myeloma with new agents. Pol Merkur Lekarski 31(186): 372-377, 2011.

43 Tan Q, Wang W, Long Y and Chen G: Therapeutic effects and associated adverse events of multikinase inhibitors in metastatic renal cell carcinoma: A meta-analysis. Exp Ther Med 9(6): 2275$2280,2015$.

44 Leung HW and Chan AL: Multikinase inhibitors in metastatic renal cell carcinoma: Indirect comparison meta-analysis. Clin Ther 33(6): 708-716, 2011.

45 Martin LG and Silva MD: Chemotherapy induced peripheral neuroparhy: A literature review. Einstein 9(4 Pt 1): 538-544, 2011.

46 Driessen CM, de Kleine-Bolt KM, Vingerhoets AJ, Mols F and Vreugdenhil G: Assesssing the impact of chemotherapy-induced peripheral neurotoxicity on the quality of life of cancer patients. Support Care Cancer 20(4): 877-881, 2012.

47 Jaggi AS and Singh N: Mechanisms in cancer-chemotherapeutic drugs-induced peripheral neuropathy. Toxicology 291(1-3): 1-9, 2012.

48 Lustberg MB, Reinbolt RE and Shapiro CL: Bone health in adult cancer survivorship. J Clin Oncol 30(30): 3665-3674, 2012.

49 Lim JS, Kim SB, Bang HY, Cheon GJ and Lee JI: High prevalence of osteoporosis in patients with gastric adenocarcinoma following gastrectomy. World J Gastroenterol 13: 6492-6497, 2007.

50 Mascia F, Mariani V, Girolomoni G and Pastore S: Blockade of the EGF receptor induces a deranged chemokine expression in keratinocytes leading to enhanced skin in ammation. Am J Pathol 163: 303-312, 2003.

51 Robert C, Soria JC, Spatz A, Le Cesne A, Malka D, Pautier P, Wechsler J, Lhomme C, Escudier B, Boige V, Armand JP and Le Chevalier T: Cutaneous side-effects of kinase inhibitors and blocking antibodies. Lancet Oncol 6: 491-500, 2005.

52 Scope A, Agero AL, Dusza SW, Myskowski PL, Lieb JA, Saltz L, Kemeny NE and Halpern AC: Randomized double-blind trial of prophylactic oral minocycline and topical tazarotene for cetuximab-associated acne- like eruption. J Clin Oncol 25: 53905396, 2007.

53 Fyfe AJ and McKay P: Toxicities associated with bleomycin. J R Coll Physicians Edinb 40(3): 213-215, 2010.

54 Perazella MA: Onco-nephrology: renal toxicities of chemotherapeutic agents. Clin J Am Soc Nephrol 7(10): 1713$1721,2012$.

55 Nam RK, Cheung P, Herschorn S, Saskin R, Su J, Klotz LH, Chang M, Kulkarni GS, Lee Y, Kodama RT and Narod SA: Incidence of complications other than urinary incontinence or erectile dysfunction after radical prostatectomy or radiotherapy for prostate cancer: A population-based cohort study. Lancet Oncol 15(2): 223-231, 2014.

56 Numico G, Longo V, Courthod G and Silvestris N: Cancer survivorship: long-term side-effects of anticancer treatments of gastrointestinal cancer. Curr Opin Oncol 27(4): 351-357, 2015.
57 Tocchetti CG, Gallucci G, Coppola C, Piscopo G, Cipresso C, Maurea C, Giudice A, Iaffaioli RV, Arra C and Maurea N: The emerging issue of cardiac dysfunction induced by antineoplastic angiogenesis inhibitors. Eur J Heart Fail 15(5): 482-489, 2013.

58 Cardinale D and Sandri MT: Role of biomarkers in chemotherapy induced cardiotoxicity. Prog Cardiovasc Dis 53: 121, 2010.

59 Scott JM, Khakoo A, Mackey JR, Haykowsky MJ, Douglas PS and Jones LW: Modulation of anthracycline-induced cardiotoxicity by aerobic exercise in breast cancer: Current evidence and underlying mechanisms. Circulation 124(5): 642-650, 2011.

60 Scott JM, Lakoski S, Mackey JR, Douglas PS, Haykowsky MJ and Jones LW: The potential role of aerobic exercise to modulate cardiotoxicity of molecularly targeted cancer therapeutics. Oncologist 18(2): 221-231, 2013.

61 Matteucci P, Carlo-Stella C, Di Nicola M, Magni M, Guidetti A, Marchesi $M$ and Gianni AM: Topical prophylaxis of conjunctivitis induced by high-dose cytosine arabinoside. Haematologica 91(2): 255-257, 2006.

62 Garcia DO and Thomson CA: Physical activity and cancer survivorship. Nutr Clin Pract 29(6): 768-779, 2014.

63 Stull VB, Snyder DC and Demark-Wahnefried W: Lifestyle interventions in cancer survivors: Designing programs that meet the needs of this vulnerable and growing population. J. Nutr 137: 243S-248S, 2007.

64 James EL, Stacey FG, Chapman K, Boyes AW, Burrows T, Girgis A, Asprey G, Bisquera A and Lubans DR: Impact of a nutrition and physical activity intervention (ENRICH: Exercise and Nutrition Routine Improving Cancer Health) on health behaviors of cancer survivors and careers: A pragmatic randomized controlled trial. BMC Cancer 15: 710, 2015.

65 Lynch BM: Sedentary behavior and cancer: a systematic review of the literature and proposed biological mechanisms. Cancer Epidemiol Biomarkers Pre 19: 2691-2709, 2010.

66 Hershman DL, Lacchetti C, Dworkin RH, Lavoie Smith EM, Bleeker J, Cavaletti G, Chauhan C, Gavin P, Lavino A, Lustberg MB, Paice J, Schneider B, Smith ML, Smith T, Terstriep S, Wagner-Johnston N, Bak K, Loprinzi CL and American Society of Clinical Oncology: Prevention and management of chemotherapy-induced peripheral neuropathy in survivors of adult cancers: American Society of Clinical Oncology clinical practice guideline. J Clin Oncol 32(18): 1941-1967, 2014.

67 Brown J, Su Y, Nelleson D, Shankar P and Mayo C: Management of epidermal growth factor receptor inhibitorassociated rash: A systematic review. J Community Support Oncol 14: 21-28, 2016.

68 Petrelli F, Borgonovo K, Cabiddu M, Coinu A, Ghilardi M, Lonati V and Barni S: Antibiotic prophylaxis for skin toxicity induced by anti-EGFR agents: A systematic review and metaanalysis. Br J Dermatol 175(6): 1166-1174, 2016.

69 Petrelli F, K Borgonovo and Barni S: Preventing or treating antiEGFR related skin rash with antibiotics? Ann Transl Med 4(16): 312, 2016. 